\title{
MINERALOGY AND GEOCHEMISTRY IMPACT ON MIDDLE AND LATE EOCENE CARBONATE ROCKS., EAST HELWAN AREA, EGYPT
}

\author{
Ibrahim, A.M. ${ }^{1}$, Kamel, S.A. ${ }^{1}$ and Abd El-Aziz, M.A. ${ }^{2}$ \\ ${ }^{I}$ Geology Dept., Fac. Sciences, Al-Azhar Univ. Cairo, Egypt \\ ${ }^{2}$ Operation Manger- Lafarge Company- Egypt
}

\section{ABSTRACT}

The present study deals with Geochemistry and Mineralogical impact on Middle and Late Eocene rocks exposed at East Helwan area -Egypt. The studied area lies between Latitudes $29^{\circ} 47^{\prime} 30^{\prime \prime}$ and $29^{\circ} 53$ ' $00^{\prime \prime} \mathrm{N}$. and longitudes $31^{\circ} 21^{\prime} 04^{\prime \prime}$ and $31^{\circ} 28^{\prime} 45^{\prime \prime} \mathrm{E}$.

Middle and Late Eocene sediments are subdivided into five rock units from base to top: Middle Eocene (Gebel Houf and Observatory Formations); Late Eocene (EL-Qurn, Wadi Garawi and Wadi Houf Formations).

Mineral composition was done on nineteen carbonate samples (using x-ray diffraction method. The study reveals the presence of a number of carbonate minerals; calcite, dolomite, and non-carbonate minerals name Gypsum, Anhydrite, Halite, and quartz in variable amounts.

Chemical composition of the carbonate rocks for fifty four samples (major and trace elements) represent Middle and Late Eocene carbonates were done. The data reveal that Middle and Late Eocene carbonates were deposited under shallow, alkaline and oxidizing environmental conditions.

\section{INTRODUCTION}

The study area lies between Latitudes $29^{\circ}$ $47^{\prime} 30^{\prime \prime}$ and $29^{\circ} 53^{\prime} 00^{\prime \prime} \mathrm{N}$. and longitudes $31^{\circ}$ $21^{\prime} 04^{\prime \prime}$ and $31^{\circ} 28^{\prime} 45^{\prime \prime}$ E. (Fig, 1 and 2). Middle and Late Eocene outcrops in the examined localities range from Lutetian to Priabonian in age.

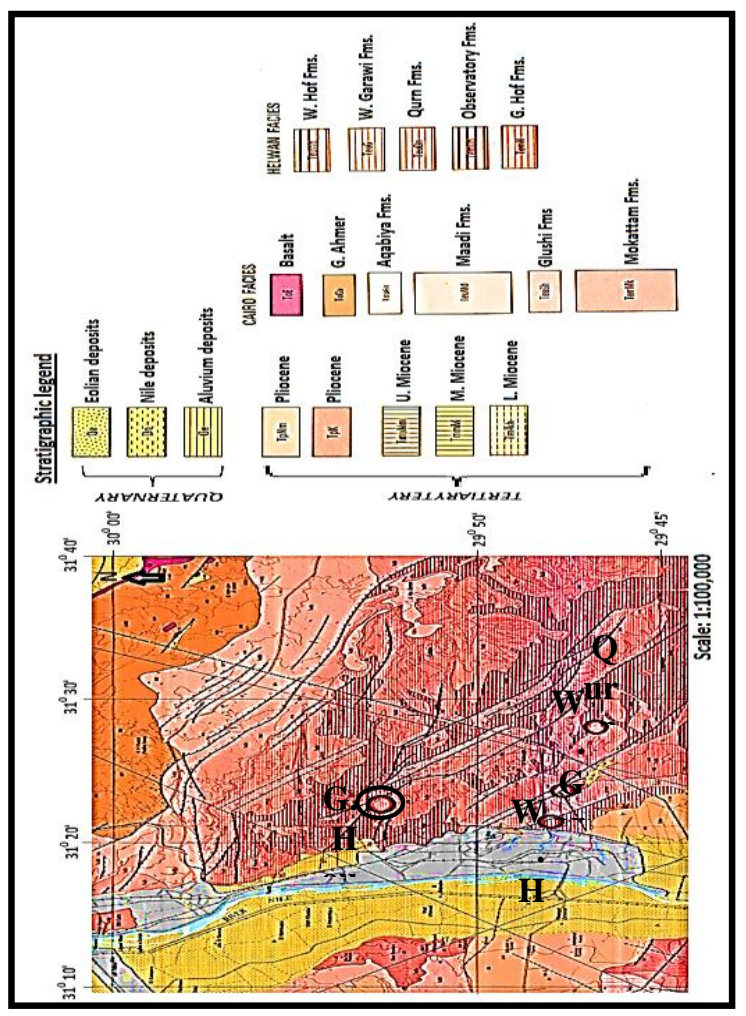

Fig. (1): Geological map of the study area (geological survey of Egypt, 1983).
Middle and Late Eocene sediments are sub divided into five rock units Eocene (Gebel Houf and Observatory Formations); Late Eocene (EL-Qurn, Wadi Garawi and Wadi Houf Formations).

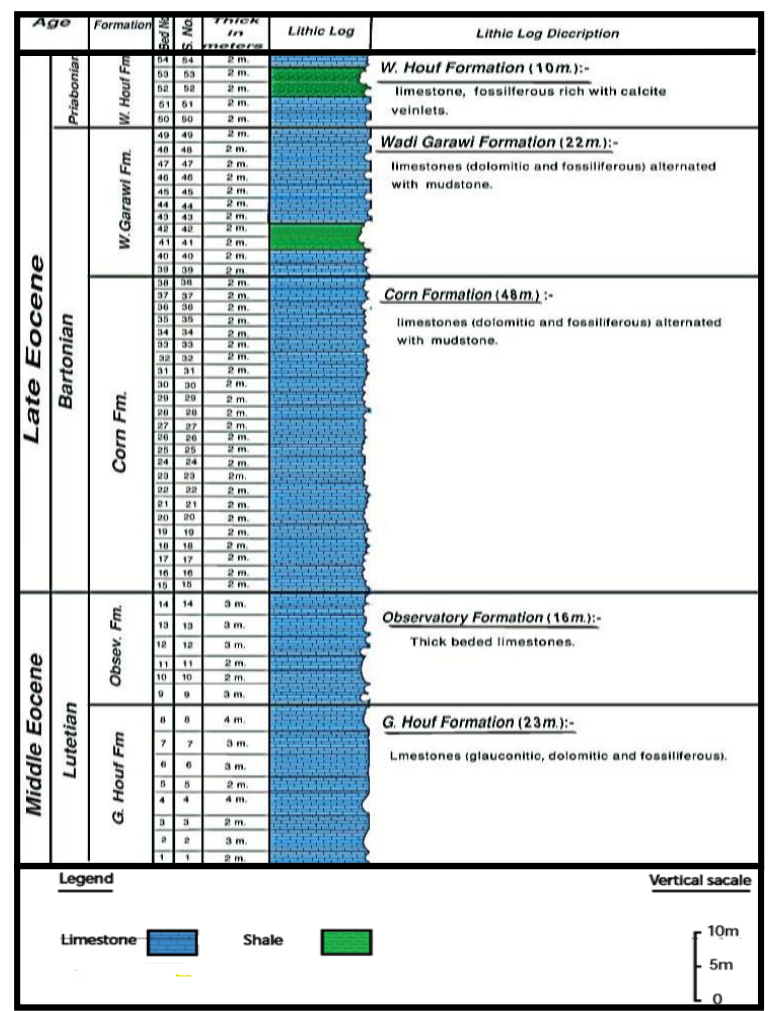

Fig. (2): Idealized composite columnar lithological section of Middle and Late Eocene rock units (East Helwan area). 


\section{MATERIALS AND METHODS OF STUDY}

Nineteen carbonate samples were analyzed, using x-ray diffraction method. The samples were selected in order to represent the carbonates of various ages, whereas samples were ground to 200 meshes and analyzed without any chemical treatment (bulk sample).

The x-ray diffraction analysis was carried out at (Egyptian Mineral Authority Labs) using the Philips X-ray diffraction (Monochromatic, $X^{\prime}$ Pert PRO type) with Ni-filter Cu-radiation, $(\chi$ $=1.542 \AA$ ) at 50 K.V., 40 M.A. and a normal scanning speed of $0.02 \mathrm{o} / \mathrm{sec}$. was used. The $\mathrm{x}$ ray diffraction analysis of the bulk limestone samples (e).

Forty six samplesanalyzed; at Lafarge Cement Company Labs; representing, Middle and Late Eocene carbonates distributed as follows: - Middle Eocene (Lutetian) G. Houf Fm., (8 samples) and Observatory Fm., (6 samples). Late Eocene (Bartonian) Qurn Fm., (21 samples) and W. Garawi Fm., (8samples). Late Eocene (Priabonian) W. Houf Fm. (3samples) for major elements $\left(\mathrm{SiO}_{2}, \mathrm{Al}_{2} \mathrm{O}_{3}\right.$, $\mathrm{Fe}_{2} \mathrm{O}_{3}, \mathrm{CaO}, \mathrm{MgO}, \mathrm{Na}_{2} \mathrm{O}, \mathrm{K}_{2} \mathrm{O}, \mathrm{P}_{2} \mathrm{O}_{5} \mathrm{SO}_{3}, \mathrm{Cl}$, L.O.I and soluble chlorides $\mathrm{Cl}-$ ) and trace elements namely ( $\mathrm{Ti}, \mathrm{Mn}, \mathrm{Ni}, \mathrm{Ba}, \mathrm{Cu}$ and $\mathrm{Sr}$ ) were quantitatively estimated using the atomic absorption method.

\section{Mineral composition}

The present study was done aiming to determine the variations and differences in the mineralogical composition of the different Middle and Late Eocene carbonate sediments represented in the study area and its significance in the environmental interpretations. The x-ray diffraction analysis of the bulk limestone samples (Table 1) detected the presence of a number of carbonate minerals; calcite, dolomite, and non-carbonate minerals namely gypsum, Halite, in addition to quartz in variable amounts. Generally, Middle and Late Eocene environments of deposition were shallow, alkaline and oxidizing environment, whereas marine basin close to the landmass that supplied the basin by evaporates, and quartz minerals.

\section{Chemical composition:- Weathering indices}

Weathering means the approach to equilibrium of a system involving rocks, air and water, and the agents of chemical weathering namely moisture, free oxygen, carbon dioxide, organic acids and nitrogen acids (Krauskopf, 1979). The indices are based on the principle that the ratio between concentrations of mobile and immobile elements should decrease over time as leaching progresses.In the present study, weathering indexproposedby Ibrahim et al., (2017) (modified after Krauskopf 1979) was used to classify Middle and Late Eocene limestone's and to correlate facies changes.

Weathering index $=$

$$
\frac{\mathrm{CaO}+\mathrm{MgO}+\mathrm{Na}_{2} \mathrm{O}+\mathrm{K}_{2} \mathrm{O}}{\mathrm{SiO}_{2}+\mathrm{Al}_{2} \mathrm{O}_{3}+\mathrm{Fe}_{2} \mathrm{O}_{3}+\mathrm{TiO}_{2}+\mathrm{CaO}+\mathrm{MgO}_{\mathrm{Na}} \mathrm{O}+\mathrm{K}_{2} \mathrm{O}}
$$

The computed weathering index for Middle and Late Eocene carbonates are shown in Table (2). The weathering index values are high fraction (except for Late Eocene (W. Garawi and W. Houf Fms.) carbonates suggesting that the studied rocks are slightly affected by the agents of weathering.

The low value for Late Eocene (W. Garawi and W. Houf Fms.) carbonates is mostly due to the predominate clastic materials rich in $\mathrm{SiO}_{2}$, $\mathrm{Al}_{2} \mathrm{O}_{3}$ and $\mathrm{Fe}_{2} \mathrm{O}_{3}$.

\section{Abundance and distribution of major components:}

\section{Oxides forming silicates:}

The chemical composition, range and the average contents of $\mathrm{SiO}_{2}, \mathrm{Al}_{2} \mathrm{O}_{3}, \mathrm{Na}_{2} \mathrm{O}$ and $\mathrm{K}_{2} \mathrm{O}$ of the different rock units are shown in Tables (3-8) and Figures (3-8). Middle Eocene, Lutetian, (G. Houf and observatory Fms) oxides forming silicate minerals are considerably low. However, regarding to the values of $\mathrm{Al}_{2} \mathrm{O}_{3}$ relative to those of $\mathrm{SiO}_{2}$, Middle Eocene limestone are characterized by the presence of free quartz, Late Eocene Bartonian (Qurn and W. Garawi Fms.) limestone's are characterized by abundant of free quartz. However, Late Eocene Priabonian (W. Houf Fms.) contain a considerable amount of free quartz. Generally it was noticed that 
there is an increase in the oxides forming silicate minerals from Middle Eocene, Lutetian, (observatory Fm) to Late Eocene Bartonian (Qurn and W. Garawi Fms.) towards Late Eocene Priabonian (W. Houf Fm.) Limestone. The inconsistent distribution of $\mathrm{Al}_{2} \mathrm{O}_{3}$ and $\mathrm{Na}_{2} \mathrm{O}$ and occasionally $\mathrm{K}_{2} \mathrm{O}$ suggests their presence don't in the form of alumina-silicate minerals.

\section{Silicon dioxide:}

The distribution of the silica content during Middle and Late Eocene times limestone reveals that there is an increasing (from Middle Eocene, observatory Fm., towards Late Eocene ,W. Houf Fm., )with decreasing in age of the limestone. The presence of free quartz suggests shallow marine conditions. The higher silica

Table (1):X-ray diffraction data of of Middle and Late Eocene carbonate samples.

\begin{tabular}{|c|c|c|c|c|c|c|c|c|c|c|c|c|c|}
\hline \multicolumn{2}{|c|}{ Age } & \multirow{10}{*}{ 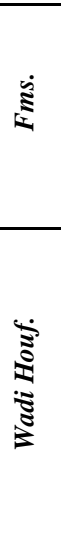 } & \multirow{4}{*}{ 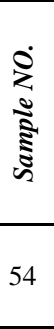 } & 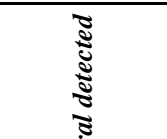 & \multirow{2}{*}{$\begin{array}{c}2 \emptyset \\
26.86 \\
\end{array}$} & \multirow{2}{*}{$\begin{array}{c}I / I_{0} \\
81.65\end{array}$} & \multirow{2}{*}{$\begin{array}{c}\boldsymbol{d} \boldsymbol{A}^{\boldsymbol{0}} \\
3.31\end{array}$} & \multirow{2}{*}{$\begin{array}{c}2 \varnothing \\
\\
39.68 \\
\end{array}$} & \multirow{2}{*}{$\begin{array}{c}I / I_{0} \\
13.80\end{array}$} & \multirow{2}{*}{$\begin{array}{l}\boldsymbol{d} \boldsymbol{A}^{\boldsymbol{0}} \\
2.27\end{array}$} & \multirow{2}{*}{$\begin{array}{c}2 \varnothing \\
\\
23.29\end{array}$} & \multirow{2}{*}{$\begin{array}{c}I / I_{0} \\
6.26\end{array}$} & \multirow{2}{*}{$\begin{array}{c}\boldsymbol{d} \boldsymbol{A}^{\boldsymbol{\theta}} \\
\\
3.81 \\
\end{array}$} \\
\hline & & & & Quartz & & & & & & & & & \\
\hline \multirow{36}{*}{ 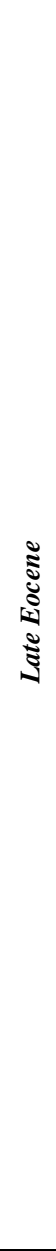 } & \multirow{8}{*}{ 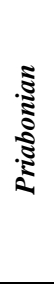 } & & & Calcite & 29.66 & 100 & 3.01 & 39.68 & 13.80 & 2.27 & 43.42 & 12.99 & 2.08 \\
\hline & & & & Albite & 39.68 & 13.08 & 2.27 & 43.42 & 12.99 & 2.08 & 48.78 & 11.84 & 1.86 \\
\hline & & & \multirow{3}{*}{51} & Calcite & 29.57 & 100 & 3.02 & 39.60 & 12.51 & 2.27 & 48.69 & 12.40 & 1.87 \\
\hline & & & & Quartz & 39.60 & 12.51 & 2.27 & 26.77 & 8.78 & 3.32 & 20.98 & 6.93 & 4.23 \\
\hline & & & & Albite & 39.60 & 12.51 & 2.27 & 48.69 & 12.40 & 1.87 & 43.34 & 11.29 & 2.08 \\
\hline & & & \multirow{3}{*}{49} & Calcite & 29.65 & 100 & 3.01 & 39.65 & 15.38 & 2.27 & 47.77 & 14.11 & 1.90 \\
\hline & & & & Clinoptilolite & 29.65 & 100 & 3.01 & 43.39 & 13.97 & 2.08 & 14.95 & 8.86 & 5.92 \\
\hline & & & & Halite & 32.04 & 8.27 & 2.79 & 56.82 & 2.20 & 1.62 & 45.63 & 1.05 & 1.98 \\
\hline & \multirow{6}{*}{ 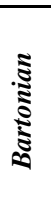 } & \multirow{6}{*}{$\begin{array}{l}\tilde{\vdots} \\
\vdots \\
\vdots \\
\vdots \\
\vdots \\
\vdots \\
\vdots\end{array}$} & & Calcite & 29.59 & 100 & 3.01 & 39.58 & 14.76 & 2.27 & 47.69 & 13.88 & 1.90 \\
\hline & & & 44 & Halite & 31.80 & 5.72 & 2.81 & 45.60 & 2.42 & 1.98 & 57.62 & 7.50 & 1.60 \\
\hline & & & & Kaolinite & 9.98 & 29.10 & 8.85 & 39.58 & 14.76 & 2.27 & 47.69 & 13.88 & 1.90 \\
\hline & & & & Calcite & 29.56 & 100 & 3.02 & 39.58 & 12.94 & 2.27 & 47.73 & 12.15 & 1.90 \\
\hline & & & 39 & Clinoptilolite & 29.56 & 100 & 3.02 & 39.58 & 12.94 & 2.27 & 31.82 & 12.08 & 2.81 \\
\hline & & & & Halite & 31.82 & 12.08 & 2.81 & 45.55 & 3.09 & 1.99 & 56.65 & 1.08 & 1.62 \\
\hline & & & & Gypsum & 29.31 & 18.73 & 3.04 & 23.58 & 11.61 & 3.77 & 31.31 & 10.81 & 2.85 \\
\hline & & & 36 & Quartz & 11.82 & 100 & 7.48 & 50.51 & 1.67 & 1.80 & 39.61 & 0.74 & 2.27 \\
\hline & & & & Calcite & 29.31 & 18.73 & 3.04 & 29.63 & 4.52 & 3.01 & 43.49 & 2.70 & 2.08 \\
\hline & & & 31 & Calcite & 29.57 & 100 & 3.02 & 39.58 & 14.12 & 2,27 & 43.31 & 13.29 & 2.08 \\
\hline & & & 28 & Calcite & 29.60 & 100 & 3.01 & 43.33 & 15.35 & 2.08 & 48.69 & 14.06 & 1.86 \\
\hline & & & & Calcite & 29.61 & 100 & 3.01 & 39.62 & 13.11 & 2.27 & 48.73 & 11.45 & 1.86 \\
\hline & & & 26 & Quartz & 39.62 & 13.11 & 2.27 & 26.81 & 5.07 & 3.32 & 57.62 & 4.89 & 1.59 \\
\hline & & & & Dolomite & 30.86 & 3.15 & 2.89 & 48.73 & 11.45 & 1.86 & 57.62 & 4.89 & 1.59 \\
\hline & & & & Calcite & 29.60 & 100 & 3.01 & 48.70 & 12.14 & 1.86 & 43.35 & 11.21 & 2.08 \\
\hline & & & & Halite & 29.60 & 100 & 3.01 & 39.58 & 13.06 & 2.27 & 56.76 & 1.79 & 1.62 \\
\hline & 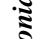 & $\Sigma$ & 24 & Quartz & 26.81 & 8.91 & 3.32 & 31.86 & 6.12 & 2.80 & 57.57 & 4.49 & 1.60 \\
\hline & $\frac{2}{2}$ & $\Xi$ & & Albite & 39.58 & 13.06 & 2.27 & 48.70 & 12.14 & 1.86 & 43.35 & 11.21 & 2.08 \\
\hline & & & & Dolomite & 28.05 & 1.13 & 3.18 & 30.98 & 2.91 & 2.88 & 48.70 & 12.14 & 1.86 \\
\hline & & & 21 & Calcite & 29.57 & 100 & 3.02 & 39.57 & 13.55 & 2.27 & 43.30 & 12.60 & 2.08 \\
\hline & & & 21 & Halite & 45.60 & 0.75 & 1.98 & 31.59 & 1,65 & 2.83 & 45.60 & 0.75 & 1.99 \\
\hline & & & & Calcite & 29.60 & 100 & 3.01 & 48.72 & 17.50 & 1.86 & 39.61 & 16.91 & 2.27 \\
\hline & & & 20 & Halite & 31.85 & 9.94 & 2.80 & 45.58 & 7.31 & 1.98 & 56.71 & 2.36 & 1.62 \\
\hline & & & & Quartz & 39.61 & 16.91 & 2.27 & 26.78 & 7.52 & 3.32 & 57.63 & 4.84 & 1.59 \\
\hline & & & 19 & Calcite & 29.58 & 100 & 3.01 & 39.57 & 14.20 & 2.27 & 48.68 & 13.92 & 1.87 \\
\hline & & & & Calcite & 29.60 & 100 & 3.01 & 39.61 & 13.15 & 2.27 & 43.35 & 12.26 & 2.08 \\
\hline & & & 15 & Quartz & 39.61 & 13.15 & 2.27 & 57.60 & 4.33 & 1.60 & 26.80 & 2.80 & 3.32 \\
\hline & & & & Dolomite & 30.89 & 6.05 & 2.89 & 48.70 & 9.57 & 1.86 & 56.82 & 1.96 & 1.62 \\
\hline & & & 12 & Dolomite & 30.88 & 100 & 2.89 & 41.05 & 12.28 & 2.19 & 44.84 & 8.33 & 2.02 \\
\hline & & 5 & 12 & Quartz & 50.83 & 7.32 & 1.79 & 50.22 & 5.78 & 1.81 & 26.76 & 3.28 & 3.33 \\
\hline & & ลั & & Calcite & 29.58 & 100 & 3.02 & 36.13 & 10.33 & 2.48 & 47.27 & 12.45 & 1.90 \\
\hline ฮั & & 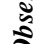 & 11 & Gypsum & 39.56 & 13.86 & 2.27 & 43.33 & 12.33 & 2.08 & 48.63 & 12.32 & 1.87 \\
\hline 10 &. & & 10 & Calcite & 29.56 & 100 & 39.56 & 13.86 & 2.27 & 2.27 & 43.31 & 13.08 & 2.08 \\
\hline$\stackrel{\Xi}{\approx}$ & క్ & & & Calcite & 29.65 & 100 & 3.01 & 36.26 & 11.47 & 2.47 & 39.68 & 14.91 & 2.27 \\
\hline 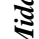 & & & 3 & Halite & 31.95 & 4.52 & 2,80 & 56,86 & 2.31 & 1.61 & 57.68 & 4.35 & 1.59 \\
\hline & & $\mathbb{Z}$ & & Dolomite & 30.99 & 1.85 & 2.88 & 48.74 & 9.92 & 1.86 & 57.68 & 4.35 & 1.59 \\
\hline & & $\dot{0}$ & 1 & Calcite & 23.22 & 7.73 & 3.82 & 29.58 & 100 & 3.01 & $39 . .61$ & 1.96 & 2.82 \\
\hline & & & 1 & Gypsum & 11.77 & 4.81 & 7.51 & 20.79 & 0.53 & 4.27 & 43.35 & 11.27 & 2.08 \\
\hline
\end{tabular}


Table (2): Weathering index values of Middle and Late Eocene Carbonates.

\begin{tabular}{|c|c|c|c|c|c|c|c|c|c|c|c|}
\hline \multicolumn{2}{|c|}{ Age } & Fms. & $\mathrm{CaO}$ & $\mathrm{MgO}$ & $\mathrm{Na}_{2} \mathrm{O}$ & $\mathbf{K}_{2} \mathbf{O}$ & $\mathrm{SiO}_{2}$ & $\mathbf{A l}_{2} \mathbf{O}_{3}$ & $\mathrm{Fe}_{2} \mathrm{O}_{3}$ & $\mathrm{TiO}_{2}$ & $\begin{array}{l}\text { Weathering } \\
\text { Index ratio }\end{array}$ \\
\hline \multirow{3}{*}{ 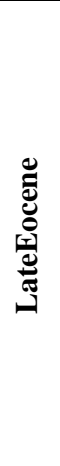 } & 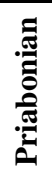 & $\begin{array}{l}\stackrel{\Xi}{\vec{B}} \\
\dot{B}\end{array}$ & 45.25 & 0.71 & 0.10 & 0.09 & 11.89 & 1.85 & 1.07 & 0.11 & 0.76 \\
\hline & \multirow{2}{*}{ فี } & 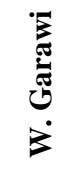 & 42.69 & 1.54 & 0.20 & 0.02 & 10.25 & 2.23 & 0.97 & 0.11 & 0.77 \\
\hline & & $\tilde{\Xi}$ & 48.26 & 1.18 & 0.77 & 0.09 & 3.91 & 1.51 & 0.55 & 0.08 & 0.89 \\
\hline \multirow{2}{*}{ 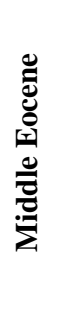 } & \multirow{2}{*}{ } & 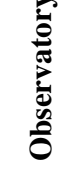 & 50.35 & 3.33 & 0.07 & 0.01 & 0.90 & 0.46 & 0.03 & 0.01 & 0.97 \\
\hline & & نُق & 50.98 & 1.13 & 0.73 & 0.03 & 1.74 & 0.81 & 0.27 & 0.03 & 0.94 \\
\hline
\end{tabular}

Table (3): chemical analyses (Major components in Wt. \%) of Middle Eocene (Lutetian , G. Houf Fm.) limestone's.

\begin{tabular}{|c|c|c|c|c|c|c|c|c|c|c|c|c|c|}
\hline \multicolumn{2}{|c|}{ 胥 } & 节 & S.No & $\underset{\%}{\mathrm{SiO}_{2}}$ & $\underset{\%}{\mathbf{A l}_{2} \mathbf{O}_{3}}$ & $\begin{array}{c}\mathrm{Fe}_{2} \mathbf{O}_{3} \\
\%\end{array}$ & $\begin{array}{c}\mathrm{CaO} \\
\%\end{array}$ & $\underset{\%}{\mathrm{MgO}}$ & $\begin{array}{c}\mathrm{Na}_{2} \mathrm{O} \\
\%\end{array}$ & $\begin{array}{c}\mathbf{K}_{\mathbf{2}} \mathbf{O} \\
\%\end{array}$ & $\begin{array}{l}\mathrm{Cl} \\
\%\end{array}$ & $\begin{array}{c}\mathrm{SO}_{3} \\
\%\end{array}$ & $\begin{array}{c}\text { L.O.I } \\
\%\end{array}$ \\
\hline \multirow{9}{*}{ 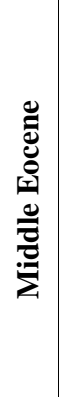 } & \multirow{9}{*}{ 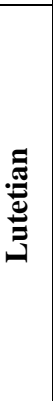 } & \multirow{9}{*}{ 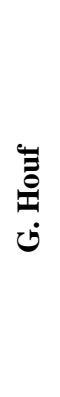 } & H8 & 1.45 & 0.71 & 0.13 & 50.71 & 1.13 & 0.03 & 0.01 & 0.11 & 2.43 & 43.32 \\
\hline & & & H7 & 1.05 & 0.72 & 0.11 & 51.17 & 0.71 & 0.91 & 0.03 & 0.10 & 3.06 & 42.13 \\
\hline & & & H6 & 1.21 & 0.85 & 0.16 & 52.33 & 0.49 & 0.04 & 0.01 & 1.71 & 0.46 & 43.07 \\
\hline & & & H5 & 1.52 & 0.55 & 0.19 & 52.03 & 1.71 & 0.39 & 0.03 & 0.20 & 0.45 & 42.05 \\
\hline & & & H4 & 1.95 & 0.71 & 0.25 & 49.23 & 1.54 & 0.61 & 0.03 & 1.48 & 1.31 & 43.20 \\
\hline & & & H3 & 1.97 & 0.70 & 0.25 & 50.39 & 1.55 & 0.59 & 0.03 & 0.83 & 1.31 & 42.91 \\
\hline & & & H2 & 1.93 & 0.82 & 0.39 & 55.21 & 0.84 & 0.22 & 0.02 & 0.02 & 0.33 & 40.47 \\
\hline & & & H1 & 2.81 & 1.44 & 0.69 & 46.78 & 1.06 & 3.08 & 0.07 & 0.05 & 1.72 & 41.90 \\
\hline & & & Average & 1.74 & 0.81 & 0.27 & \begin{tabular}{|l|}
50.98 \\
\end{tabular} & 1.13 & 0.73 & 0.03 & 0.56 & 1.38 & 42.38 \\
\hline
\end{tabular}

content than those given by Pettijohn (1975) 0.7 to $7.91 \%$, can be attributed to the presence of $\mathrm{SiO}_{2}$ in the form of quartz grains which were microscopically identified.

Corbel (1959) proved that there is an increase in $\mathrm{SiO}_{2}$ content towards the warmer climatic zone while Dekimpe et al. (1961) noted that with increasing $\mathrm{pH}$ there is a decrease in the silica content.
Accordingly Middle and Late Eocene limestone were mostly deposited under relatively warm alkaline conditions. However the $\mathrm{pH}$ degree of alkalinity during Middle Eocene, G. Houf Fm. (Less in $\mathrm{SiO}_{2}$ content) was higher than that prevailed during Middle and Late Eocene time. 
Table (4): chemical analyses(Major components in Wt. \%) of Middle Eocene (Lutetian, observatory Fm.) limestone's

\begin{tabular}{|c|c|c|c|c|c|c|c|c|c|c|c|c|c|}
\hline \multirow[b]{2}{*}{ 뮌 } & \multirow{2}{*}{\multicolumn{2}{|c|}{ 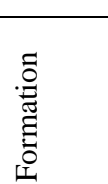 }} & \multirow[b]{2}{*}{ S.No } & \multirow[b]{2}{*}{$\begin{array}{c}\mathrm{SiO}_{2} \\
\%\end{array}$} & \multirow[b]{2}{*}{$\underset{\%}{\mathrm{Al}_{2} \mathrm{O}_{3}}$} & \multirow[b]{2}{*}{$\begin{array}{c}\mathrm{Fe}_{2} \mathrm{O}_{3} \\
\%\end{array}$} & \multirow[b]{2}{*}{$\begin{array}{c}\mathrm{CaO} \\
\%\end{array}$} & \multirow[b]{2}{*}{$\underset{\%}{\mathrm{MgO}}$} & \multirow[b]{2}{*}{$\underset{\%}{\mathrm{Na}_{2} \mathrm{O}}$} & \multirow[b]{2}{*}{$\begin{array}{c}\mathrm{K}_{2} \mathrm{O} \\
\%\end{array}$} & \multirow[b]{2}{*}{$\mathrm{Cl} \%$} & \multirow[b]{2}{*}{$\begin{array}{c}\mathrm{SO}_{3} \\
\%\end{array}$} & \\
\hline & & & & & & & & & & & & & $\begin{array}{c}\text { L.O.I } \\
\%\end{array}$ \\
\hline \multirow{7}{*}{ 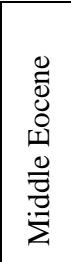 } & \multirow{7}{*}{ 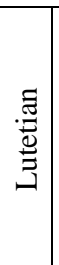 } & \multirow{7}{*}{ 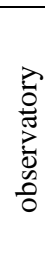 } & H-14 & 0.51 & 0.42 & 0.01 & 56.39 & 0.59 & 0.03 & 0.01 & 0.06 & 2.07 & 40.69 \\
\hline & & & $\mathrm{H}-13$ & 0.71 & 0.31 & 0.02 & 50.63 & 0.45 & 0.04 & 0.02 & 0.04 & 5.31 & 42.76 \\
\hline & & & $\mathrm{H}-12$ & 1.94 & 0.16 & 0.05 & 36.22 & 17.76 & 0.16 & 0.01 & 0.06 & 0.75 & 42.76 \\
\hline & & & $\mathrm{H}-11$ & 0.72 & 0.55 & 0.05 & 49.48 & 0.32 & 0.05 & 0.02 & 0.08 & 2.18 & 45.76 \\
\hline & & & $\mathrm{H}-10$ & 0.71 & 0.74 & 0.03 & 54.77 & 0.39 & 0.06 & 0.01 & 0.31 & 0.80 & 42.45 \\
\hline & & & H-9 & 0.81 & 0.55 & 0.04 & 54.62 & 0.44 & 0.06 & 0.01 & 0.12 & 0.58 & 43.10 \\
\hline & & & Average & 0.90 & 0.46 & 0.03 & 50.35 & 3.33 & 0.07 & 0.01 & 0.11 & 1.95 & 42.92 \\
\hline
\end{tabular}

Table (5):- chemical analyses(Major components in Wt. \%) of Late Eocene (Bartonian, Qurn Fm.)limestone's

\begin{tabular}{|c|c|c|c|c|c|c|c|c|c|c|c|c|c|}
\hline \multicolumn{2}{|l|}{ 岁 } & $\begin{array}{l}\frac{c}{0} \\
\frac{0}{\pi} \\
\frac{5}{2} \\
\text { o }\end{array}$ & S.No & $\begin{array}{c}\mathrm{SiO}_{2} \\
\%\end{array}$ & $\begin{array}{c}\mathrm{Al}_{2} \mathrm{O}_{3} \\
\%\end{array}$ & $\begin{array}{c}\mathrm{Fe}_{2} \mathrm{O}_{3} \\
\%\end{array}$ & $\begin{array}{c}\mathrm{CaO} \\
\%\end{array}$ & $\begin{array}{c}\text { MgO } \\
\%\end{array}$ & $\begin{array}{c}\mathrm{Na}_{2} \mathrm{O} \\
\%\end{array}$ & $\mathrm{~K}_{2} \mathrm{O} \%$ & $\begin{array}{l}\mathrm{Cl} \\
\%\end{array}$ & $\begin{array}{c}\mathrm{SO}_{3} \\
\%\end{array}$ & $\begin{array}{c}\text { L.O.I } \\
\%\end{array}$ \\
\hline \multirow{22}{*}{ 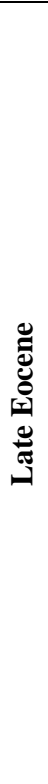 } & \multirow{22}{*}{ 苞 } & \multirow{22}{*}{ 돌 } & Q -35 & 1.38 & 0.84 & 0.10 & 52.02 & 1.81 & 0.25 & 0.03 & 0.21 & 0.43 & 42.96 \\
\hline & & & Q -34 & 1.28 & 0.37 & 0.28 & 53.75 & 0.43 & 0.04 & 0.02 & 1.35 & 0.73 & 41.99 \\
\hline & & & Q -33 & 1.45 & 0.66 & 0.18 & 52.41 & 0.43 & 0.38 & 0.03 & 0.55 & 3.89 & 40.23 \\
\hline & & & Q -32 & 4.64 & 1.08 & 0.46 & 40.01 & 0.55 & 1.55 & 0.11 & 2.40 & 25.70 & 23.26 \\
\hline & & & Q -31 & 1.88 & 0.56 & 0.12 & 54.02 & 0.49 & 0.17 & 0.02 & 0.58 & 0.32 & 42.46 \\
\hline & & & Q -30 & 1.38 & 0.56 & 0.07 & 53.09 & 0.40 & 0.12 & 0.01 & 0.59 & 0.11 & 43.33 \\
\hline & & & Q -29 & 2.18 & 0.43 & 0.08 & 53.81 & 0.41 & 0.09 & 0.03 & 0.12 & 0.06 & 42.96 \\
\hline & & & Q -28 & 1.72 & 0.81 & 0.17 & 53.83 & 0.48 & 0.11 & 0.02 & 0.25 & 0.03 & 43.27 \\
\hline & & & Q - 27 & 3.60 & 1.47 & 0.42 & 50.46 & 0.70 & 0.36 & 0.04 & 0.79 & 0.26 & 41.97 \\
\hline & & & Q -26 & 4.14 & 1.66 & 0.50 & 48.37 & 2.22 & 0.31 & 0.03 & 0.88 & 0.16 & 41.88 \\
\hline & & & Q -25 & 8.18 & 1.78 & 0.65 & 45.94 & 0.65 & 1.26 & 0.20 & 2.17 & 0.09 & 39.29 \\
\hline & & & Q - 24 & 10.08 & 2.07 & 0.85 & 42.66 & 1.23 & 0.88 & 0.28 & 1.47 & 0.13 & 39.80 \\
\hline & & & Q - 23 & 3.78 & 2.38 & 1.05 & 46.31 & 2.08 & 1.02 & 0.33 & 1.75 & 0.13 & 41.17 \\
\hline & & & Q -22 & 3.35 & 2.68 & 0.91 & 44,08 & 2.16 & 1.40 & 0.12 & 3.64 & 0.20 & 41,46 \\
\hline & & & Q -21 & 3.37 & 3.53 & 1.33 & 40.31 & 1.01 & 4.75 & 0.23 & 7.44 & 0.99 & 37,04 \\
\hline & & & Q -20 & 9.68 & 3.07 & 1.01 & 41.60 & 1.36 & 1.93 & 0.11 & 3.86 & 0.17 & 37.57 \\
\hline & & & Q -19 & 2.03 & 0.81 & 0.20 & 50.71 & 0.54 & 0.24 & 0.02 & 1.51 & 0.32 & 42.49 \\
\hline & & & Q -18 & 6.06 & 1.01 & 0.51 & 48.62 & 1.25 & 0.21 & 0.02 & 0.78 & 0.29 & 40.98 \\
\hline & & & Q -17 & 3.60 & 2.48 & 0.82 & 47.03 & 1.56 & 0.25 & 0.03 & 1.67 & 0.11 & 42.44 \\
\hline & & & Q -16 & 3.98 & 3.06 & 1.31 & 45.56 & 2.97 & 0.54 & 0.09 & 0.91 & 0.16 & 41.42 \\
\hline & & & Q -15 & 4.40 & 0.95 & 0.64 & 48.83 & 2.12 & 0.29 & 0.03 & 0.87 & 0.21 & 41.91 \\
\hline & & & Average & 3.91 & 1.51 & 0.55 & 48.26 & 1.18 & 0.77 & 0.09 & 1.61 & 1.64 & 40.47 \\
\hline
\end{tabular}

Table (6):-chemical analyses(Major components in Wt. \%) of Late Eocene (Bartonian ,W. Garawi Fm.)limestone's

\begin{tabular}{|c|c|c|c|c|c|c|c|c|c|c|c|c|c|}
\hline \multicolumn{2}{|c|}{ 岁 } & 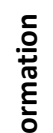 & S.No & $\begin{array}{c}\mathrm{SiO}_{2} \\
\%\end{array}$ & $\begin{array}{c}\mathrm{Al}_{2} \mathrm{O}_{3} \\
\%\end{array}$ & $\begin{array}{c}\mathrm{Fe}_{2} \mathrm{O}_{3} \\
\%\end{array}$ & $\begin{array}{c}\mathrm{CaO} \\
\%\end{array}$ & $\begin{array}{c}\text { MgO } \\
\%\end{array}$ & $\begin{array}{c}\mathrm{Na}_{2} \mathrm{O} \\
\%\end{array}$ & $\begin{array}{c}\mathrm{K}_{2} \mathrm{O} \\
\%\end{array}$ & $\begin{array}{l}\mathrm{Cl} \\
\%\end{array}$ & $\begin{array}{c}\mathrm{SO}_{3} \\
\%\end{array}$ & $\begin{array}{c}\text { L.O.I } \\
\%\end{array}$ \\
\hline \multirow{9}{*}{ 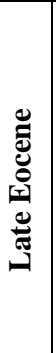 } & \multirow{9}{*}{ 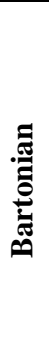 } & \multirow{9}{*}{ 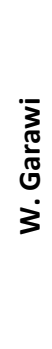 } & W.G- 49 & 3.77 & 1.21 & 0.46 & 46.65 & 1.20 & 0.41 & 0.04 & 1.07 & 9.91 & 35.40 \\
\hline & & & W.G- 48 & 6.83 & 1.69 & 0.73 & 45.85 & 1.97 & 0.10 & 0.01 & 1.25 & 0.94 & 41.22 \\
\hline & & & W.G -47 & 10.62 & 2.12 & 0.98 & 41.17 & 2.67 & 0.09 & 0.02 & 2.41 & 0.15 & 41.55 \\
\hline & & & W.G- 46 & 6.15 & 1.33 & 0.59 & 46.51 & 1.83 & 0.02 & 0.01 & 2.01 & 0.03 & 42.43 \\
\hline & & & W.G-45 & 6.52 & 1.69 & 0.58 & 45.81 & 2.12 & 0.03 & 0.01 & 1.34 & 0.14 & 42.26 \\
\hline & & & W.G- 44 & 20.55 & 4.02 & 1.78 & 35.53 & 1.12 & 0.60 & 0.05 & 2.00 & 0.85 & 34.69 \\
\hline & & & W.G- 43 & 18.07 & 4.15 & 1.92 & 37.07 & 1.00 & 0.26 & 0.03 & 1.87 & 0.19 & 36.38 \\
\hline & & & W.G- 39 & 9.50 & 1.63 & 0.68 & 42.90 & 2.25 & 0.08 & 0.01 & 3.83 & 0.17 & 41.59 \\
\hline & & & Average & 10.25 & 2.23 & 0.97 & 42.69 & 1.54 & 0.20 & 0.02 & 1.97 & 1.55 & 39.32 \\
\hline
\end{tabular}


IBRAHIM, A.M., et al.

Table (7): Chemical composition (Major components in Wt. \%) of LateEocene (Priabonian, Wadi Houf Fm.)limestone's

\begin{tabular}{|c|c|c|c|c|c|c|c|c|c|c|c|c|c|}
\hline \multicolumn{2}{|l|}{ 嵌 } & है & S.No & $\begin{array}{c}\mathrm{SiO}_{2} \\
\%\end{array}$ & $\begin{array}{c}\mathrm{Al}_{2} \mathrm{O}_{3} \\
\%\end{array}$ & $\begin{array}{c}\mathrm{Fe}_{2} \mathrm{O}_{3} \\
\%\end{array}$ & $\mathrm{CaO} \%$ & $\begin{array}{c}\mathrm{MgO} \\
\%\end{array}$ & $\begin{array}{c}\mathrm{Na}_{2} \mathrm{O} \\
\%\end{array}$ & $\begin{array}{c}\mathrm{K}_{2} \mathrm{O} \\
\%\end{array}$ & $\begin{array}{l}\mathrm{Cl} \\
\%\end{array}$ & $\mathrm{SO}_{3}$ & L.O.I \\
\hline \multirow{3}{*}{ 罢 } & \multirow{3}{*}{ 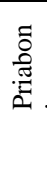 } & \multirow{3}{*}{ 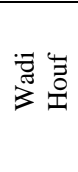 } & W.H 54 & 17.00 & 2.36 & 1.26 & 41.70 & 0.74 & 0.12 & 0.10 & 0.75 & 0.02 & 36.17 \\
\hline & & & W.H 51 & 12.13 & 1.74 & 0.87 & 45.56 & 0.71 & 0.11 & 0.12 & 0.19 & 0.09 & 38.27 \\
\hline & & & W.H 50 & 6.55 & 1.45 & 1.09 & 48.48 & 0.67 & 0.06 & 0.04 & 0.63 & 0.13 & 41.31 \\
\hline
\end{tabular}

\section{Aluminum Oxide:}

According to Krauskopf (1956) and Wey (1961), $\mathrm{Al}_{2} \mathrm{O}_{3}$ is more soluble in acidic medium than $\mathrm{SiO}_{2}$ and in neutral medium $(5-6 \mathrm{pH})$ $\mathrm{Al}_{2} \mathrm{O}_{3}$ is insoluble whereas $\mathrm{SiO}_{2}$ retains its solubility. In alkaline medium the two solubility's meet and increase together $(\mathrm{pH}$ over
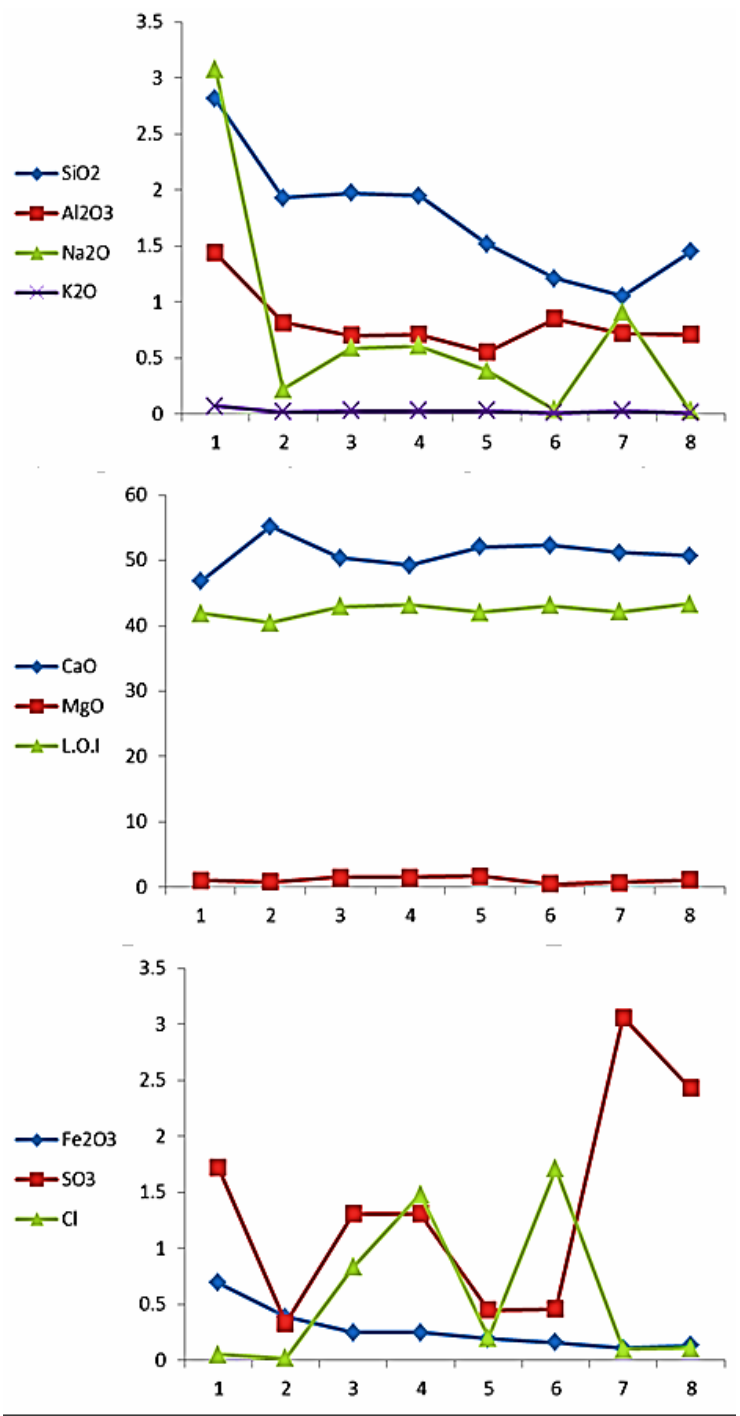

Figure (3): Distribution curves of chemical components \%) of Middle Eocene( G. Houf Fm.) limeston
9). The consistent distribution of both $\mathrm{Al}_{2} \mathrm{O}_{3}$ and $\mathrm{SiO}_{2}$ favoured their contemporaneous deposition suggesting that the $\mathrm{pH}$ of the medium was 7.8 to 8 .
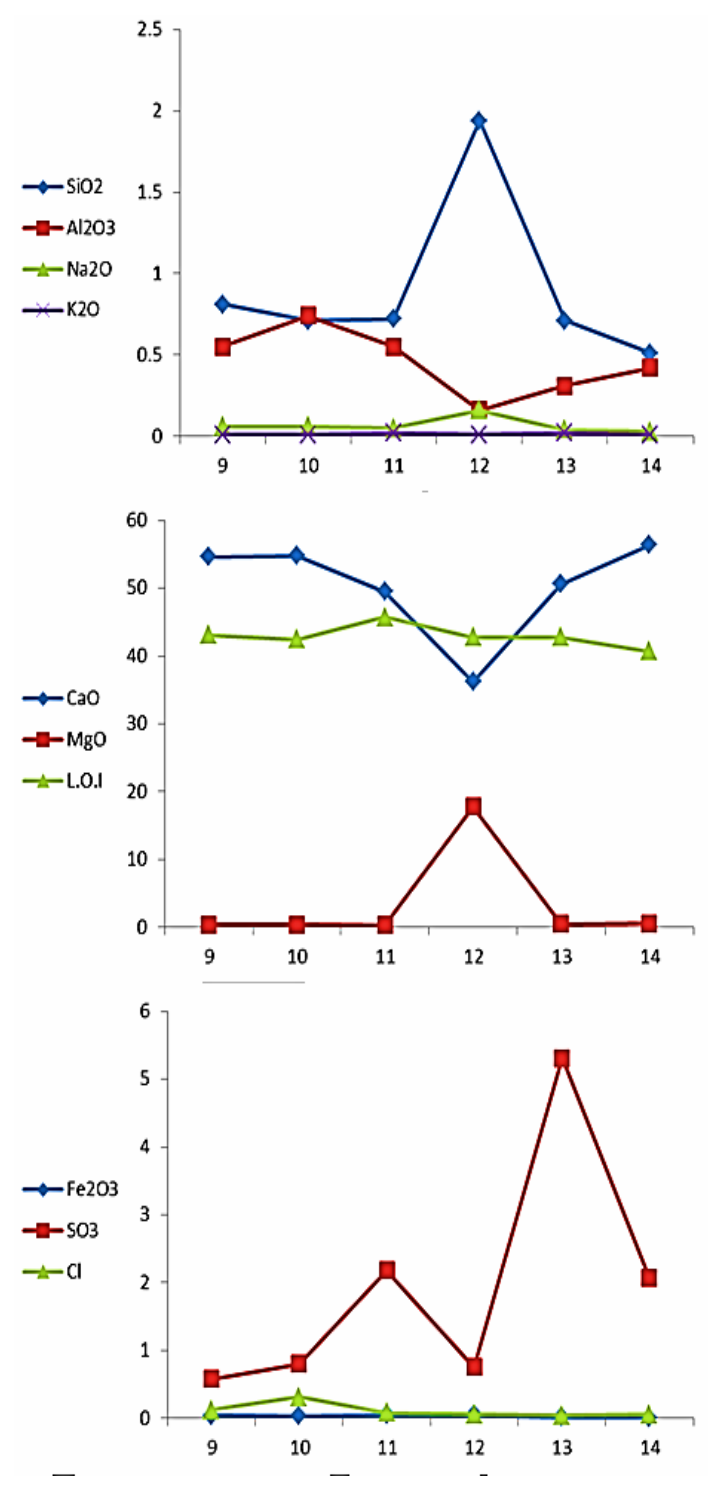

Figure (4): Distribution curves of chemical components (in wt. \%) of Middle Eocene (observatory Fm.) limestone. 
MINERALOGY AND GEOCHEMISTRY IMPACT ON MIDDLE ...

\begin{tabular}{|c|c|c|c|c|c|c|c|c|c|c|c|c|c|c|c|}
\hline & & fidd & Eo & & & & & & Lat & Eoc & sene & & & & \\
\hline & & & tia & & & & & $\mathrm{Ba}$ & tonia & & & Pri & abol & ian & \\
\hline & Ho & & Obs & $\mathbf{n a}$ & & & Qur & & w. & farr: & & &. $\mathrm{Ho}$ & & Fms \\
\hline $\begin{array}{l}\text { 营 } \\
\text { 总 } \\
\text { 恕 }\end{array}$ & 常 & 貢 & $\begin{array}{l}\text { 党 } \\
\text { 怘 } \\
\text { tg }\end{array}$ & 蛋 & 菅 & $\begin{array}{l}\text { 荵 } \\
\text { 怘 } \\
\text { 㤎 }\end{array}$ & 蛋 & 帠 & $\begin{array}{l}\text { 胥 } \\
\text { 惡 } \\
\text { 茄 }\end{array}$ & 桑 & 胥 & 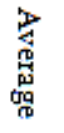 & 蛋 & 貢 & 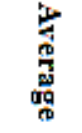 \\
\hline$\stackrel{\sim}{a}$ & $\begin{array}{l}N \\
\infty \\
\infty\end{array}$ & $\overrightarrow{\theta_{1}}$ & : & $\vec{b}$ & 它 & w & $\begin{array}{l}\vec{\circ} \\
:\end{array}$ & 岕 & 总 & 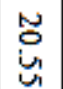 & $\dot{\vec{u}}$ & 宫 & 겅 & 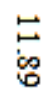 & \& \\
\hline $\begin{array}{l}\circ \\
\infty \\
0\end{array}$ & $\stackrel{\overrightarrow{+}}{+}$ & 兑 & $\begin{array}{l}\text { ㅇ } \\
\text { 占 }\end{array}$ & 움 & 온 & $\overrightarrow{0}$ & w & 怘 & 点 & $\stackrel{\vec{v}}{\vec{v}}$ & 㟢 & 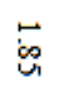 & $\underset{⿱ 宀}{\infty}$ & 它 & 일 \\
\hline 号 & : & 웁 & 啬 & : & 웅 & 总 & 岕 & :웅 & 8 & 宫 & 염 & 家 & 悹 & 客 & 。㐌 \\
\hline $\begin{array}{l}\text { U. } \\
\text { : } \\
0\end{array}$ & $\begin{array}{l}\underset{\mathrm{H}}{\mathrm{W}} \\
\mathrm{\omega}\end{array}$ & $\underset{\infty}{\stackrel{a}{a}}$ & 㝕 & 岁 & 岕 & $\begin{array}{l}c \\
\infty \\
\omega \\
\alpha\end{array}$ & 岕 & $\begin{array}{l}\text { 总 } \\
\text { 웅 }\end{array}$ & $\begin{array}{l}\text { 芯 } \\
\text { 㤩 }\end{array}$ & 拿 & 岕 & 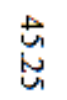 & $\begin{array}{c}+\infty \\
\stackrel{\infty}{\infty} \\
\stackrel{\infty}{\infty}\end{array}$ & 䓠 & $\therefore$ \\
\hline$\vec{\omega}$ & $\stackrel{\sim}{-2}$ & $\begin{array}{l}\text { 足 } \\
\text { 占 }\end{array}$ & 岕 & 倣 & 怘 & $\underset{\infty}{\stackrel{5}{*}}$ & 孞 & 足 & $\overrightarrow{\breve{t}}$ & $\stackrel{N}{a}$ & : & 엄 & $\underset{i}{0}$ & 임 & s。學 \\
\hline 엏 & 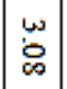 & : & 웅 & 웅 & 잉 & 업 & $\stackrel{t}{u}$ & 웅 & 옹 & 足 & 웅 & :응 & 움 & 용 & 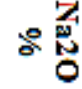 \\
\hline $\begin{array}{l}8 \\
8 \\
0\end{array}$ & 号 & 웅 & 웅 & 웅 & 웅 & : & 品 & 웅 & 웅 & : & 웅 & : & 운 & 웅 & 。祭 \\
\hline : & $\underset{⿱ ⺌}{-}$ & 怘 & $\stackrel{ }{\circ}$ & 完 & 응 & $\stackrel{\vec{\alpha}}{\sigma}$ & $\stackrel{+}{+}$ & $\stackrel{ }{\stackrel{6}{*}}$ & $\vec{b}$ & 虫 & 客 & 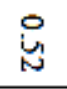 & 언 & $\stackrel{ }{\circ}$ & \& \\
\hline$\underset{\omega}{\omega_{\infty}}$ & به & 孞 & $\overrightarrow{0}$ & 岕 & 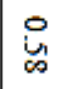 & $\vec{a}$ & 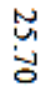 & : & $\overrightarrow{u_{u}}$ & 吕 & : & : & 운 & 웅 & $\therefore 8$ \\
\hline 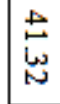 & 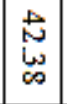 & $\begin{array}{l}\text { 㕝 } \\
\text { 点 }\end{array}$ & 点 & 岕 & 㕝 & $\begin{array}{l}\text { 点 } \\
\text { 品 }\end{array}$ & 志 & \begin{tabular}{l}
\multirow{\omega}{\omega}{} \\
\multirow{2}{*}{}
\end{tabular} & $\begin{array}{l}\text { 岕 } \\
\text { 岕 }\end{array}$ & 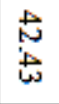 & $\begin{array}{l}\stackrel{w}{u} \\
\stackrel{\Delta}{\Delta}\end{array}$ & $\begin{array}{l}w_{\infty} \\
\infty \\
W_{1}\end{array}$ & $\underset{\omega}{\stackrel{E}{\omega}}$ & $\stackrel{\text { a }}{\stackrel{\omega}{a}}$ & \& \\
\hline
\end{tabular}

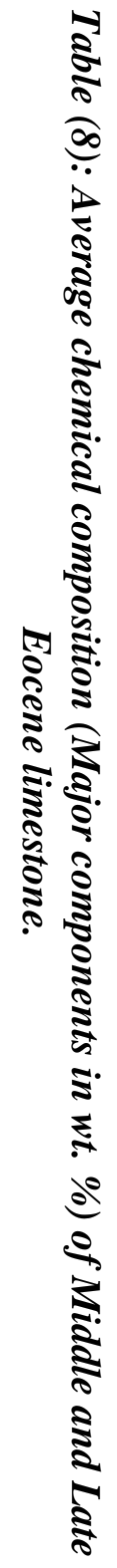

Table (9): Average Ca/Mg ratio in Middle and Late Eocene limestone

\begin{tabular}{|c|c|c|c|c|c|}
\hline \multirow{2}{*}{ Age } & \multicolumn{2}{|c|}{ Middle Eocene } & \multicolumn{3}{c|}{ Late Eocene } \\
\hline Fms & G. Houf & observatory & Qurn & W. Garawi & W. Houf \\
\hline $\mathrm{Ca}$ & 36.2 & 35.7 & 34.3 & 30.3 & 32.1 \\
\hline $\mathrm{Mg}$ & 0.68 & 2.0 & 0.71 & 0.92 & 0.43 \\
\hline $\mathrm{Ca} / \mathrm{Mg}$ Ratio & 53.24 & 17.85 & 48.3 & 32.93 & 74.65 \\
\hline
\end{tabular}




\section{Iron oxides:}

The distribution of ferric oxide in Middle and Late Eocene carbonates (Table 4) shows to a great extent consistent distribution with those of both $\mathrm{SiO}_{2}$ and $\mathrm{Al}_{2} \mathrm{O}_{3}$ suggesting the presence of $\mathrm{Fe}_{2} \mathrm{O}_{3}$ connected with silt or clay fractions in The variation in $\mathrm{Fe}_{2} \mathrm{O}_{3}$ concentration among Middle and Late Eocene carbonates (Table 4 and fig.3) can be attributed agreement with Martens (1939). Millot (1970) stated that "during diagenesis iron has a great tendency to re-enter silicate structure. Castano and Garrels (1950) stated that" the residence time for iron and alumina in sea in years is very short. Hence it should expect that iron oxides, silica and alumina are to be concentrated in continental and near-shore marine environments". to variation in the environment of deposition. However the $\mathrm{pH}$ degree of alkalinity during Middle Eocene, W.Houf Fm.(Less in $\mathrm{Fe}_{2} \mathrm{O}_{3}$
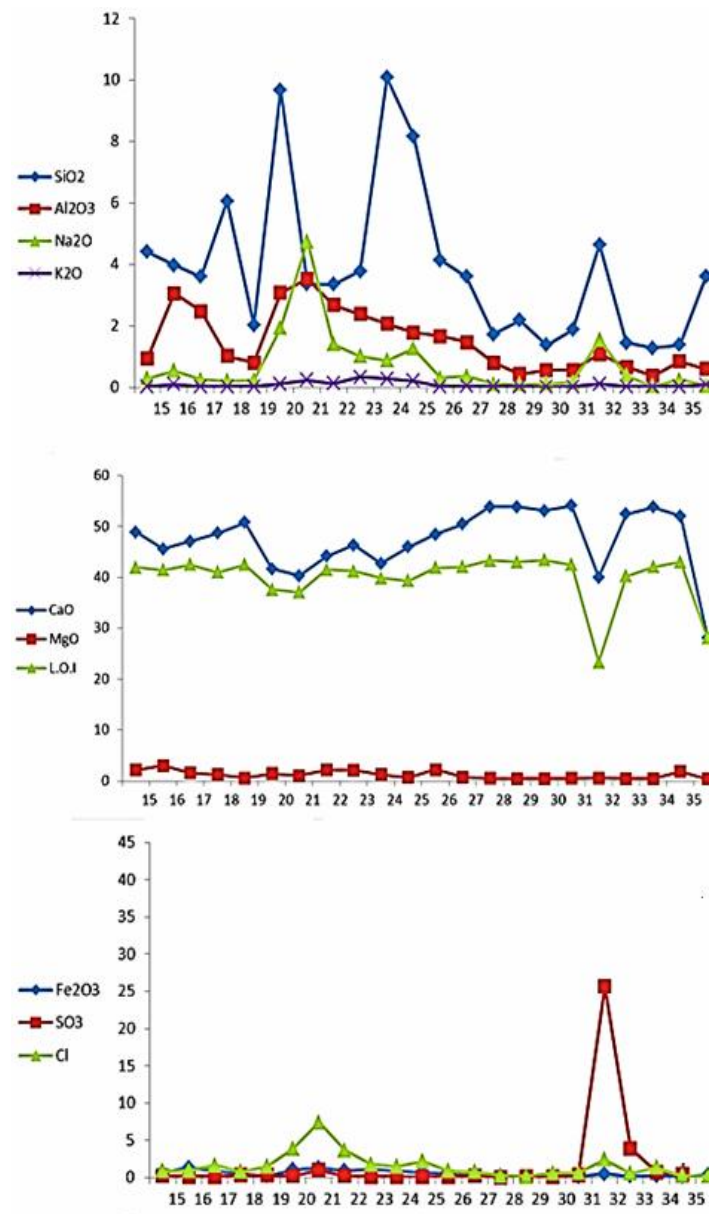

Figure (5):- Distribution curves of chemical components .(in wt. \%) of Late Eocene ( W. Garawi Fm.) limestone. was lower than that prevailed during Middle and Late Eocene time.

\section{Oxides forming carbonate Calcium Oxide:}

The distribution of $\mathrm{CaO}$ in limestones reveals that there's a decrease inCaO content with time from Middle Eocenetowards Late Eocene contrary to the silica distribution . However Trask (1939) mentioned that" the higher the salinity, the greater the content of $\mathrm{CaCO}_{3}$, this relationship is connected with higher temperature and greaterorganic production". In addition Kukal (1971) stated that" the content of natural salts and increase in temperature decrease the $\mathrm{CaCO}_{3}$ solubility and also the increased content of $\mathrm{Ca}^{2+}$ ions from other sources cause the decreased solubility of $\mathrm{CaCO}_{3}$ ". Nevertheless the higher $\mathrm{CaCO}_{3}$ content at Middle Eocene, G. and Houf Fms.) limestone seems to be due to the fact that the depth of water during deposition was not deep
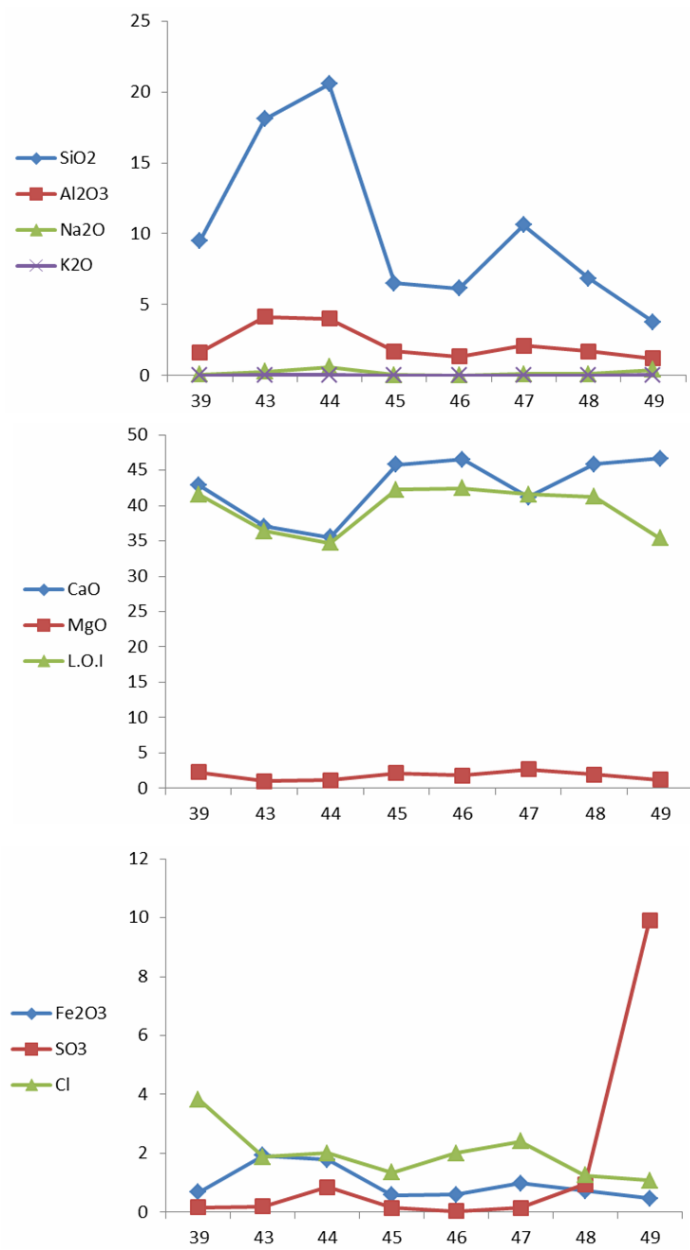

Figure (6):- Distribution curves of chemical components (in wt. \%) of Late Eocene (Qurn Fm.) limestone 
enough to cause a great variation in temperature and to increase the solubility of $\mathrm{CaCO}_{3}$ or the amounts of the argillaceous materials derived to the site of deposition were un-considerable, leading to the relative increase in the $\mathrm{CaCO}_{3}$ at the expense of $\mathrm{SiO}_{2}$ and $\mathrm{Al}_{2} \mathrm{O}_{3}$ contents.

\section{Magnesium oxide:}

The distribution of $\mathrm{MgO}$ in Middle Eocene and Late Eocene reveals that $\mathrm{MgO}$ follow $\mathrm{CaO}$ in its manner of distribution. This can be attributed to variations in the ecological and paleontological parameters of the environments under which the studied limestones were
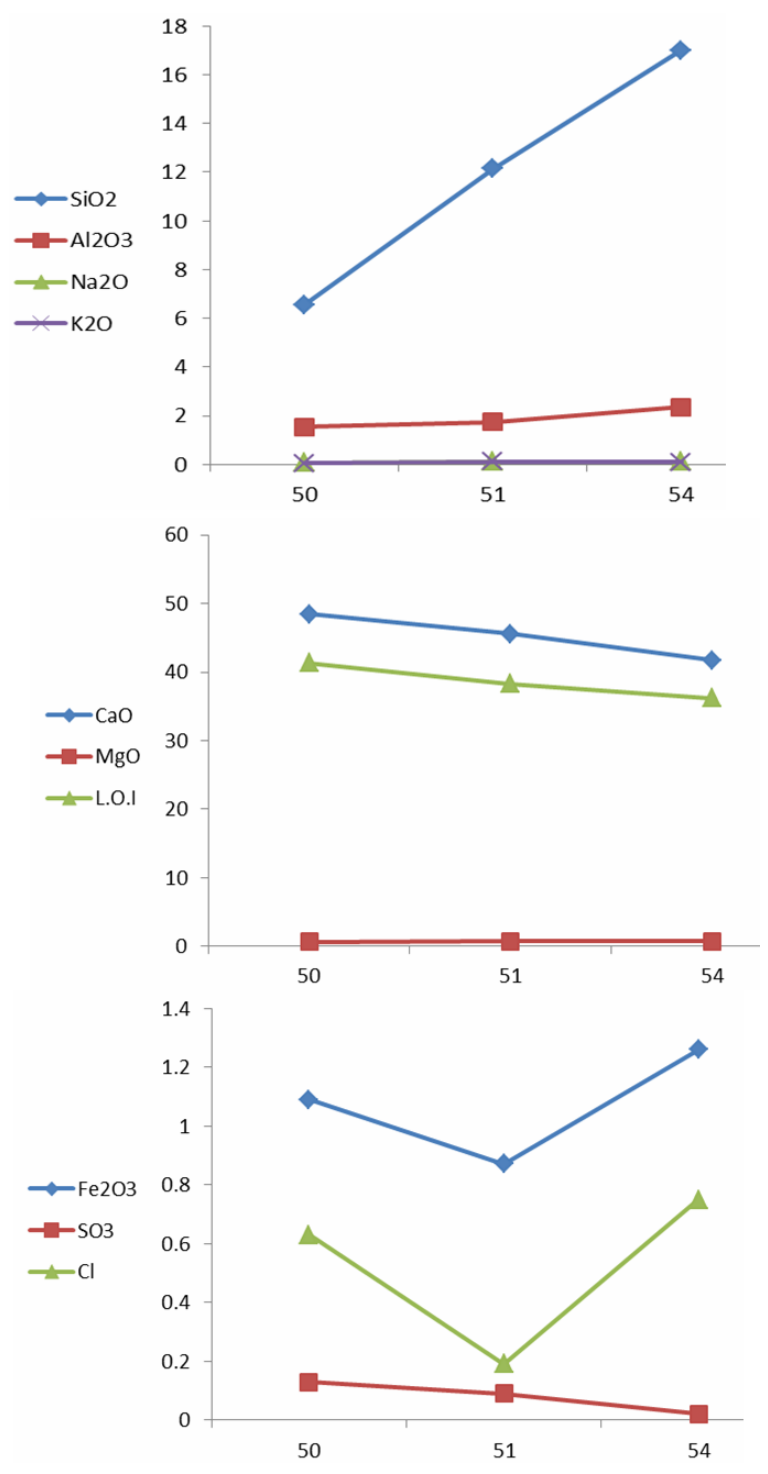

Figure (7): Distribution curves of chemical components (in wt. \%) of Middle and Late Eocene limestone deposited. Chave (1954) suggested that there is a direct relation between the content of $\mathrm{MgO}$ and temperature.

Chilingar (1963) stated that "the $\mathrm{MgO}$ content increases with salinity but is simultaneously affected by so many other factors that it cannot serve as an adequate indicator". He also noted that in carbonate sediments the $\mathrm{Ca} / \mathrm{Mg}$ ratio increases sea wards with depth. Chilingar (1967) stated that " in as muchas shallow near-shore waters are systematically warmer as arule than deep offshore waters; the gross $\mathrm{Ca} / \mathrm{Mg}$ ratio reflects temperature-depth-distance from shore."
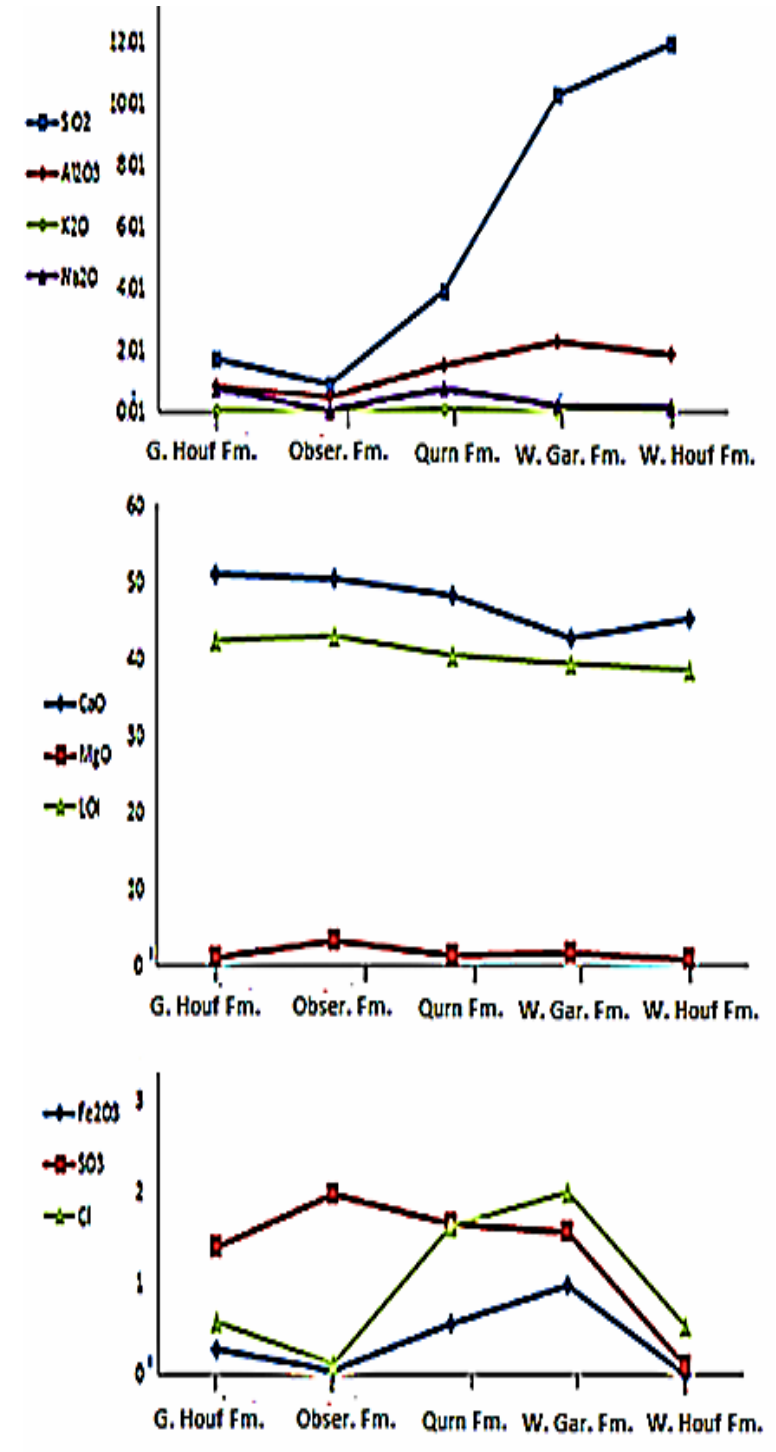

Figure (8): Distribution curves of chemical components (in wt. \%) of Late Eocene (Wadi Houf Fm.) limestone 
Thegross $\mathrm{Ca} / \mathrm{Mg}$ ratio is represented by table (10) and figure (9), suggest that there is no particular trend for the distribution of $\mathrm{Ca} / \mathrm{Mg}$ average ratio and this can beattributed to the selective adsorption of $\mathrm{Mg}^{2+}$ by illlte clays Chilingar (op.cit) so, the gross ratio reflects the illite content or perhaps the degree of diagenesis of Middle and Late Eocene limestone.

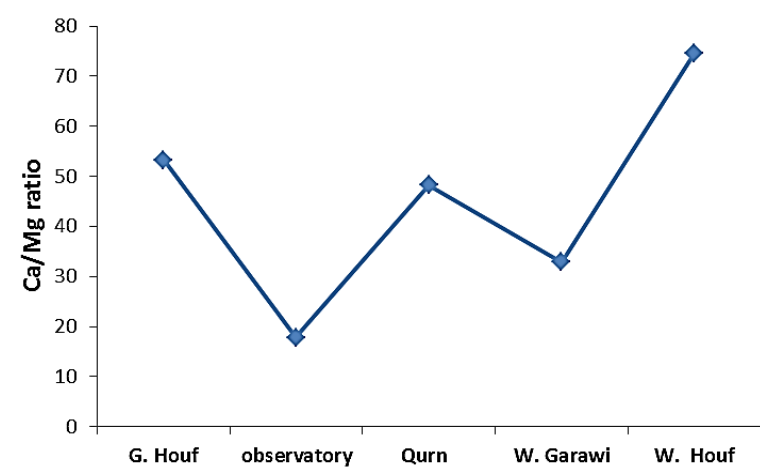

Fig. (9): Average $\mathrm{Ca} / \mathrm{Mg}$ in Middle and Late Eocene limestone

\section{Sodium and Potassium oxides:}

From table (10) and figure (10) it is obvious that $\mathrm{Na}_{2} \mathrm{O}$ predominates $\mathrm{K}_{2} \mathrm{O}$ in Middle Eocene, Lutetian, (G. Houf Fm) and Late Eocene limestones except for those of Middle Eocene, ( observatory Fm.). The outstanding characteristics of $\mathrm{K}_{2} \mathrm{O}$ in comparison with that of $\mathrm{Na}_{2} \mathrm{O}$ has been noted for a long time in a way like that described by(Noll, 1931 ; Urbain, 1933; Goldschmidt, 1937; Harvey, 1949 and Millot 1949). Milot (1949) stated that "one sees that in the course of continental weathering sodium turns out to be much more mobile than potassium and dominates the latter in natural water. Potassium is sorted up and conserved in a preferential way". Again Millot (1970) mentioned that "if one considers the behaviour of $\mathrm{K}$ ions in solution, one sees that they are preferentially adsorbed by the finegrained particles of the sediments". Consequently the slight predominance of $\mathrm{K}_{2} \mathrm{O}$ contents over $\mathrm{Na}_{2} \mathrm{O}$ contents in Middle Eocene, (observatory Fm.) limestones could be understood according to Garrels and Christ (1965) and Weaver (1967), the K/Na ratio is very important, where low ratio favour the formation of montmorillonite materials and high ratio leads to the formation of illite. It seems that clays in the form of illite predominate over montmorillonite clays in Middle Eocene, (observatory Fm.) and montmorillonite predominate over illite in Middle Eocene, Lutetian, (G. Houf Fm) and Late Eocene Formations limestones.

Table (10): Average K / Na Ratio in Middle and Late Eocene limestone

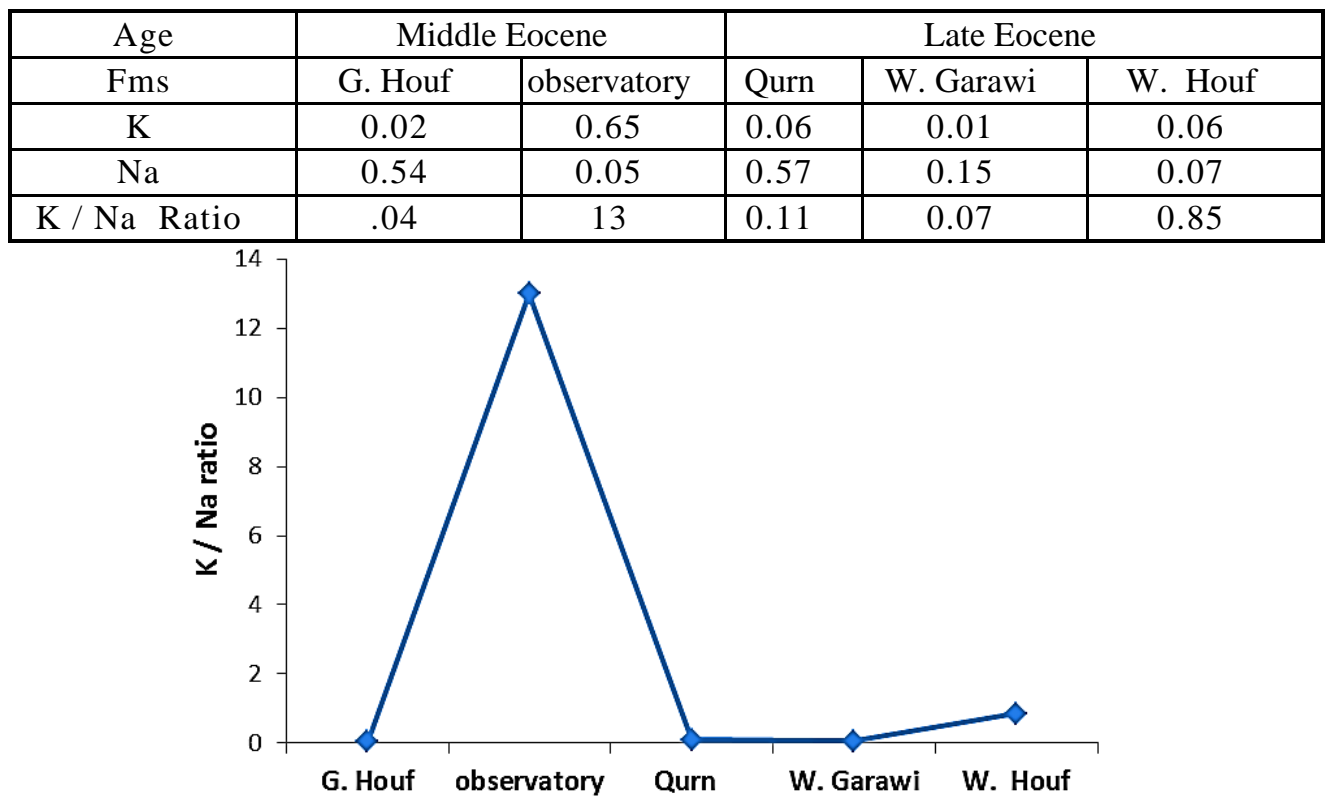

Fig. (10): Average K / Na Ratio in Middle and Late Eocene limestone. 


\section{Soluble chlorides:}

The soluble chlorides contents of Middle and Late Eocene limestones are relatively higher than that given by (Turckian and Wedepohl, 1961) $(0.015 \%)$, indicating the prevalence of warm climate as well as relatively shallow occasionally restricted medium.

\section{Abundance and distribution of trace elements:}

The major features of the distribution of trace elements in sedimentary rocks can be related to ionic size charge and bond character, Most of the trace elements are more abundant in fine-grained detrital sediments than in sandstones or carbonate rocks. notable concentrations of trace elements formed by sedimentary processes alone are not common (Krauskopf, 1979). Since the elements behave differently in their migration and deposition so, the abundance and behavior of each element in Middle and Late Eocene carbonate sediments will be considered.

\section{Titanium:}

Titanium is the most abundant trace element recorded in Middle and Late Eocene limestones. Since the average concentration of titanium in carbonates, given by Turekian and Wedepohl (1961) is 400 ppm, The lower average titanium contents were recorded in the limestone's of Middle Eocene Lutetian (observatory and G. Houf Fms.), and the heights contents in Late Eocene, Bartonian, (Qurn and W. Garawi Fms.) and in Late Eocene, Priabonian, (W. Houf Fm.) limestone's (table12). The distribution of titanium in Middle and Late Eocene limestones does not show any particular trend for distribution and this can be attributed to the different rates of sedimentation (Arrhenius, 1954).The high Ti content values recorded in Late Eocene limestones can be attributed to the amounts of terrigenous materials brought to the site deposition (Isayeva, 1977).

Table (11): chemical analyses(trace elements in Wt. \%) of Middle Eocene (Lutetian , G. Houf Fm.) limestone's

\begin{tabular}{|c|c|c|c|c|c|c|c|c|c|}
\hline & & . & S.No & TiO2 & P2O5 & MnO & SrO & $\mathrm{Cr} 2 \mathrm{O3}$ & ZnO \\
\hline & & & H8 & 0.01 & 0.03 & 0.00 & 0.09 & 0.00 & 0.00 \\
\hline & & & H7 & 0.02 & 0.04 & 0.00 & 0.12 & 0.00 & 0.00 \\
\hline & & & H6 & 0.02 & 0.05 & 0.01 & 0.08 & 0.00 & 0.01 \\
\hline$\stackrel{\square}{\circ}$ & .ే & 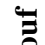 & H5 & 0.03 & 0.10 & 0.01 & 0.12 & 0.00 & 0.01 \\
\hline st & 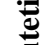 & $\stackrel{\rho}{1}$ & H4 & 0.04 & 0.14 & 0.01 & 0.14 & 0.00 & 0.02 \\
\hline 흥 & & نت & H3 & 0.04 & 0.14 & 0.01 & 0.14 & 0.00 & 0.01 \\
\hline & & & H2 & 0.04 & 0.13 & 0.02 & 0.15 & 0.00 & 0.01 \\
\hline & & & H1 & 0.05 & 0.12 & 0.05 & 0.11 & 0.00 & 0.00 \\
\hline & & & Average & 0.03 & 0.09 & 0.02 & 0.12 & n.d & 0.01 \\
\hline
\end{tabular}

Table (12): chemical analyses(trace elements in Wt. \%) of Middle Eocene (Lutetian , observatory Fm.) limestone's

\begin{tabular}{|c|c|c|c|c|c|c|c|c|c|}
\hline \multicolumn{2}{|c|}{ AGE } & Fms & S.No & TiO2 & P2O5 & MnO & SrO & $\mathrm{Cr} 2 \mathrm{O3}$ & $\mathrm{ZnO}$ \\
\hline \multirow{7}{*}{ 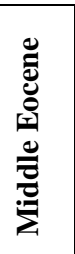 } & \multirow{7}{*}{ 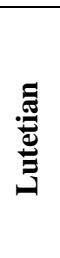 } & \multirow{7}{*}{ 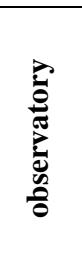 } & H-14 & 0.01 & 0.02 & 0.01 & 0.07 & 0.00 & 0.00 \\
\hline & & & H-13 & 0.01 & 0.03 & 0.00 & 0.13 & 0.00 & 0.00 \\
\hline & & & H-12 & 0.01 & 0.03 & 0.01 & 0.16 & 0.00 & 0.00 \\
\hline & & & H-11 & 0.01 & 0.03 & 0.01 & 0.06 & 0.00 & 0.01 \\
\hline & & & H-10 & 0.01 & 0.03 & 0.00 & 0.08 & 0.00 & 0.00 \\
\hline & & & H-9 & 0.01 & 0.03 & 0.01 & 0.08 & 0.00 & 0.00 \\
\hline & & & Averag & 0.01 & 0.03 & 0.01 & 0.10 & n.d & 0.01 \\
\hline
\end{tabular}


Table (13):- chemical analyses(trace elements in Wt. \%) of Late Eocene (Bartonian , Qurn Fm.)limestone's

\begin{tabular}{|c|c|c|c|c|c|c|c|c|c|}
\hline & & 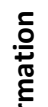 & S.No & TiO2 & P2O5 & $\mathrm{MnO}$ & SrO & Cr2O3 & $\mathrm{ZnO}$ \\
\hline & & & Q-38 & 0.04 & 0.03 & 0.00 & 0.40 & 0.01 & 0.00 \\
\hline & & & Q -37 & 0.06 & 0.03 & 0.01 & 0.29 & 0.00 & 0.00 \\
\hline & & & Q -36 & 0.06 & 0.03 & 0.01 & 0.36 & 0.00 & 0.00 \\
\hline & & & Q -35 & 0.02 & 0.05 & 0.01 & 0.17 & 0.00 & 0.00 \\
\hline & & & Q -34 & 0.02 & 0.04 & 0.03 & 0.15 & 0.00 & 0.00 \\
\hline & & & Q -33 & 0.02 & 0.03 & 0.01 & 0.15 & 0.00 & 0.00 \\
\hline & & & Q -32 & 0.07 & 0.06 & 0.01 & 0.25 & 0.00 & 0.00 \\
\hline & & & Q -31 & 0.01 & 0.03 & 0.01 & 0.11 & 0.00 & 0.01 \\
\hline & & & Q -30 & 0.01 & 0.03 & 0.01 & 0.11 & 0.00 & 0.01 \\
\hline & & & Q -29 & 0.02 & 0.05 & 0.01 & 0.11 & 0.01 & 0.00 \\
\hline & & & Q -28 & 0.02 & 0.07 & 0.01 & 0.14 & 0.00 & 0.01 \\
\hline $\bar{E}$ & . & & Q -27 & 0.05 & 0.07 & 0.01 & 0.22 & 0.01 & 0.01 \\
\hline 오 & อี & $\frac{5}{3}$ & Q-26 & 0.07 & 0.21 & 0.01 & 0.23 & 0.01 & 0.02 \\
\hline$\stackrel{\Xi}{\Xi}$ & 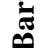 & & $Q-25$ & 0.10 & 0.07 & 0.01 & 0.18 & 0.01 & 0.01 \\
\hline & & & Q -24 & 0.12 & 0.07 & 0.01 & 0.21 & 0.01 & 0.02 \\
\hline & & & $Q-23$ & 0.16 & 0.08 & 0.02 & 0.22 & 0.01 & 0.03 \\
\hline & & & Q-22 & 0.12 & 0.09 & 0.01 & 0.19 & 0.01 & 0.01 \\
\hline & & & Q -21 & 0.19 & 0.41 & 0.01 & 0.09 & 0.01 & 0.00 \\
\hline & & & Q -20 & 0.13 & 0.16 & 0.01 & 0.11 & 0.01 & 0.01 \\
\hline & & & Q -19 & 0.03 & 0.08 & 0.01 & 0.10 & 0.01 & 0.01 \\
\hline & & & Q -18 & 0.07 & 0.08 & 0.01 & 0.25 & 0.00 & 0.02 \\
\hline & & & Q -17 & 0.11 & 0.11 & 0.01 & 0.13 & 0.01 & 0.03 \\
\hline & & & Q -16 & 0.16 & 0.17 & 0.01 & 0.17 & 0.01 & 0.03 \\
\hline & & & Q -15 & 0.08 & 0.10 & 0.02 & 0.25 & 0.01 & 0.02 \\
\hline & & & Average & 0.08 & 0.10 & 0.01 & 0.17 & 0.01 & 0.02 \\
\hline
\end{tabular}

Table (14):-chemical analyses(Major components in Wt. \%) of Late Eocene (Bartonian ,W. Garawi Fm.)limestone's

\begin{tabular}{|c|c|c|c|c|c|c|c|c|c|}
\hline \multicolumn{2}{|l|}{ 岕 } & 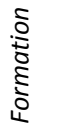 & S.No & $\mathrm{TiO} 2$ & P2O5 & $\mathrm{MnO}$ & $\mathrm{SrO}$ & $\mathrm{Cr} 2 \mathrm{O} 3$ & $\mathrm{ZnO}$ \\
\hline \multirow{9}{*}{ 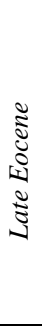 } & \multirow{9}{*}{ 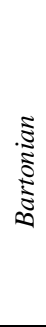 } & \multirow{9}{*}{ 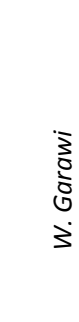 } & W.G- 49 & 0.06 & 0.08 & 0.00 & 0.43 & 0.01 & 0.00 \\
\hline & & & W.G- 48 & 0.08 & 0.11 & 0.00 & 0.33 & 0.01 & 0.00 \\
\hline & & & W.G -47 & 0.11 & 0.13 & 0.00 & 0.29 & 0.01 & 0.00 \\
\hline & & & W.G- 46 & 0.07 & 0.09 & 0.01 & 0.31 & 0.00 & 0.00 \\
\hline & & & W.G-45 & 0.07 & 0.11 & 0.00 & 0.31 & 0.00 & 0.00 \\
\hline & & & W.G- 44 & 0.19 & 0.11 & 0.03 & 0.24 & 0.01 & 0.00 \\
\hline & & & W.G- 43 & 0.20 & 0.10 & 0.04 & 0.24 & 0.01 & 0.00 \\
\hline & & & W.G- 39 & 0.07 & 0.08 & 0.01 & 0.34 & 0.01 & 0.00 \\
\hline & & & Average & 0.11 & 0.10 & 0.02 & 0.31 & 0.01 & n.d \\
\hline
\end{tabular}

Again titanium has an intermediate ionic potential, hence it is precipitated by hydrolysis in the form of hydroxides at still low alkaline $\mathrm{pH}$ values. It seems that Late Eocene limestones were deposited under alkaline conditions that permit Ti to be concentrated in Late Eocene Limestones.

\section{Phosphates:}

The distribution averages of $\mathrm{P}_{2} \mathrm{O}_{5}$ show that there is no particular trend for distribution with time.

The lower average $\mathrm{P}_{2} \mathrm{O}_{5}$ average contents were recorded in the limestones of Middle 
Eocene Lutetian (observatory and G. Fms.) (table 16), and the high contents in Late Eocene, Bartonian, (Qurn and W. Garawi Fms.) and Priabonian, (W. Houf Fm.) limestones (table 16) than the average given by Turekian and Wedepohl (op.cit) (0.04\%)reveals that Late Eocenelimestones were deposited under reduced slightly alkaline conditions (Kukal, 1971) that permit $\mathrm{P}_{2} \mathrm{O}_{5}$ to be concentrated in Late Eocene limestones.

\section{Manganese Oxide:}

The averages of manganese in Middle and Late Eocene limestones show that it is present in subordinate concentrations in comparison with that given by Turekian and Wedepohl, 1961, (1100 ppm). The subordinate manganese concentration can be attributed to the fact that manganese is less mobile under oxidizing conditions and it willbe mobilized in a reducing environment and precipitated as divalent ion in carbonates (Manheim, 1961; Wedepohl, 1964 and Hartmann, 1964). It seems that Middle and Late Eocene limestones were deposited underrelatively reduced alkaline conditions that permit $\mathrm{Mn}$ to be mobilized.

\section{Strontium Oxide:}

The distribution of Strontium in Middle and Late Eocene limestones does not show any particular trend. Chilingar (1967) stated that "Strontium increases towards shore-wards, where warm waters". Kukal (1971), Kitano and Kawasaki (1958) and Bathurst (1968) suggested that the $\mathrm{SrO}$ content is affected mainly

Table (15): Chemical composition (trace elements in Wt. \% of Late Eocene (Priabonian, Wadi Houf Fm.) limestone's.

\begin{tabular}{|c|c|c|c|c|c|c|c|c|c|}
\hline \multicolumn{2}{|l|}{ 舒 } & 兽 & S.No & TiO2 & P2O5 & MnO & SrO & $\mathrm{Cr} 2 \mathrm{O3}$ & $\mathrm{ZnO}$ \\
\hline \multirow{4}{*}{ 竎 } & \multirow{4}{*}{ 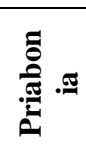 } & \multirow{4}{*}{ 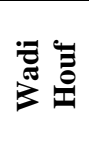 } & W.H 54 & 0.17 & 0.14 & 0.01 & 0.23 & 0.00 & 0.00 \\
\hline & & & W.H 51 & 0.09 & 0.12 & 0.01 & 0.23 & 0.00 & 0.00 \\
\hline & & & W.H 50 & 0.08 & 0.09 & 0.00 & 0.18 & 0.00 & 0.00 \\
\hline & & & Average & 0.11 & 0.11 & 0.01 & 0.21 & n.d & n.d \\
\hline
\end{tabular}

Table (16): Average Chemical composition (Major components in Wt. \%) Of Middle Eocene limestone

\begin{tabular}{|c|c|c|c|c|c|c|c|c|c|}
\hline \multicolumn{2}{|c|}{ 峁 } & 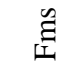 & Range & $\mathrm{TiO} 2$ & $\mathrm{P} 2 \mathrm{O} 5$ & $\mathrm{MnO}$ & $\mathrm{SrO} \mathrm{O}$ & $\mathrm{Cr} 2 \mathrm{O} 3$ & $\mathrm{ZnO}$ \\
\hline \multirow{9}{*}{ 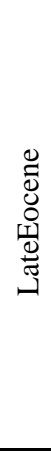 } & \multirow{3}{*}{ 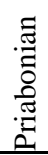 } & \multirow{3}{*}{ خ } & Min. & 0.08 & 0.09 & 0.01 & 0.18 & n.d & n.d \\
\hline & & & Max. & 0.17 & 0.14 & 0.01 & 0.23 & n.d & n.d \\
\hline & & & Average & 0.11 & 0.11 & 0.01 & 0.21 & n.d & n.d \\
\hline & \multirow{6}{*}{ 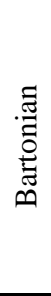 } & \multirow{3}{*}{ 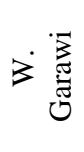 } & Min. & 0.06 & 0.08 & 0.01 & 0.24 & 0.01 & n.d \\
\hline & & & Max. & 0.20 & 0.13 & 0.04 & 0.43 & 0.01 & n.d \\
\hline & & & Average & 0.11 & 0.10 & 0.02 & 0.31 & 0.01 & n.d \\
\hline & & \multirow{3}{*}{ 砉 } & Min. & 0.01 & 0.03 & 0.01 & 0.09 & 0.01 & 0.01 \\
\hline & & & Max. & 0.19 & 0.41 & 0.03 & 0.25 & 0.01 & 0.03 \\
\hline & & & Average & 0.08 & 0.10 & 0.01 & 0.17 & 0.01 & 0.02 \\
\hline \multirow{6}{*}{ 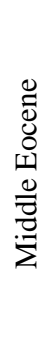 } & \multirow{6}{*}{ 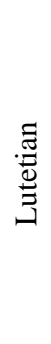 } & \multirow{3}{*}{$\begin{array}{l}\overrightarrow{0} \\
\stackrel{0}{0} \\
\sum_{0}^{2} \\
0 \\
0 \\
0\end{array}$} & Min. & 0.01 & 0.02 & 0.01 & 0.06 & n.d & 0.01 \\
\hline & & & Max. & 0.01 & 0.03 & 0.01 & 0.16 & n.d & 0.01 \\
\hline & & & Average & 0.01 & 0.03 & 0.01 & 0.10 & n.d & 0.01 \\
\hline & & \multirow{3}{*}{$\begin{array}{l}\stackrel{4}{\Xi} \\
\stackrel{0}{I} \\
ن\end{array}$} & Min. & 0.01 & 0.09 & 0.01 & 0.08 & n.d & 0.01 \\
\hline & & & Max. & 0.05 & 0.14 & 0.05 & 0.15 & n.d & 0.02 \\
\hline & & & Average & 0.03 & 0.09 & 0.02 & 0.12 & n.d & 0.01 \\
\hline
\end{tabular}


by the aragonite content and $\mathrm{SrO}$ content in carbonate sediments appear to be a reliable indicator of the salinity and temperature.

Pilkey and Godell (1963) record that differences in salinity cause greater changes in shell composition than differences in temperature. Accordingly, the high strontium content in the studied carbonates than the average given by Turekian and Wedepohl, (1961) (610ppm) suggests that the salinity and temperature of the environment was high saline and to certain extent high temperature.

\section{Chromium oxide:}

Chromium oxide did not detected in Late Eocene, Priabonian, (W.Houf Fm.) rock unit. Chromium oxide contents in Late Eocene, Bartonian, (Qurn and W. Garawi Fms.), carbonates rock units is higher than that given by Turekian and Wedepohl, (1961) (11ppm). It seems that Late Eocene, Bartonian, (Qurn and W. Garawi Fms.), carbonates rock units were deposited under more alkaline conditions that permit $\mathrm{Cr}_{2} \mathrm{O}_{3}$ to be concentrated.

\section{Zinc Oxide:}

Late Eocene Bartonian (W. Garawi Fm) and Priabonian (W. Houf Fm.) rock units, the Zinc oxide is not detected(Tables 12-17, Figs 11- 16 ).The detected Zinc Oxidecontents in Middle Eocene, Lutetian, (G. Houf and observatory Fms) and Late Eocene, Bartonian, (Qurn Fm.) is higher than that given by Turekian and Wedepohl, (1961) (20 ppm) reveal formation underrelatively reduced alkalineconditions that permit zinc oxide to be concentrated.

\section{Physiochemical Parameters}

\section{1- Temperature:}

The values of the $\mathrm{Ca} / \mathrm{Mg}$, ratio during Middle and Late Eocene rocks carbonates suggest that they are limestones relatively rich by magnesium reflecting prevalence of warm marine conditions with an occasional increase in the temperature causing the increase in the magnesium content of the precipitated calcium carbonates, since the values of $\mathrm{SiO}_{2}, \mathrm{Al}_{2} \mathrm{O}_{3}$ and $\mathrm{Fe}_{2} \mathrm{O}_{3}$, indicate that the amount of clay minerals is practically negligible.

\section{2- Salinity:}

The soluble chlorides contents during
Middle and Late Eocene times are relatively higher than that given by (Turekian and Wedepohl, 1961) $(0.015 \%)$, indicating the prevalence of warm climate as well as relatively shallow occasionally restricted medium.

\section{3- The Acidity and Alkalinity (pH):}

Middle and Late Eocene sediments consists of proper limestone beds as well as highly calcareous beds with an oscillation in the oxygen and $\mathrm{CO}_{2}$ contents at time intervals suggesting the prevalence of alkaline conditions.

\section{4- Recrystallization and dolomitization:}

Recrystallization according to Bausch (1968) or aggrading neomorphism is restricted to limestones with less than $2 \%$ clays. Generally, Middle and Late Eocene times carbonate sediments, according to Tucker's classification (1981), are clustered mainly as limestones (Less than $10 \% \mathrm{MgCO}_{3}$ ). The classification of Middle and Late Eocene carbonate sediments as limestones supports the assumption of local dolomitization and local source of magnesium ions.

\section{CONCLUSIONS}

Middle and Late Eocene sediments are subdivided into five rock units from base to top: Middle Eocene (Gebel Houf and Observatory Formations); Late Eocene (EL-Qurn, Wadi Garawi and Wadi Houf FormationsMineral composition was done on nineteen carbonate samples (using $\mathrm{x}$-ray diffraction method. The study reveals the presence of a number of carbonate minerals; calcite, dolomite, and noncarbonate minerals name Gypsum, Anhydrite, Halite, and quartz in variable amounts. Chemical composition of the carbonate rocks for fifty four samples (major and trace elements) represent Middle and Late Eocene carbonates were done. The data reveal that Middle and Late Eocene carbonates were deposited undershallow, alkaline and oxidizing environmental conditions.

\section{REFERENCES}

Arrhenius G. (1954): Origin and accumulation of alumina-silicates in the ocean. Tellus, Stockholm, v. 3, p. 215-220.

Bathurst, R.G.C. (1968): Precipitation of ooids and other aragonite fabrics in warm seas. In: G. Muller and G.M. Friedman (Editors), 'Recent 
